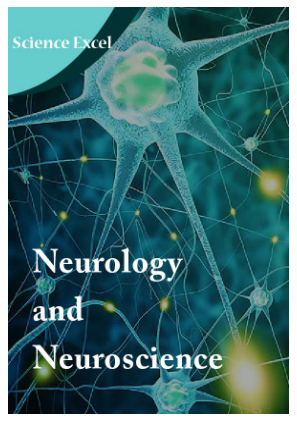

\section{*Correspondence}

Yinyun Li

School of Systems Science, Beijing Normal University, Beijing, 100875,

China, E-mail:Yinyun@bnu.edu.cn

- Received Date: 03 Apr 2020;

- Accepted Date: 26 Apr 2020;

- Publication Date: 01 May 2020.

Keywords

Synaptic Plasticity, AMPA receptors, Trafficking, Endocytosis, Exocytosis

Copyright

(C) 2020 Science Excel. This is an openaccess article distributed under the terms of the Creative Commons Attribution 4.0 International license.

\title{
Theoretical model of postsynaptic AMPA receptor trafficking during synaptic plasticity
}

\author{
Huairui Huang' and Yinyun Lí2* \\ 'School of Government Management, Beijing Normal University, Beijing, 100875, China \\ ${ }^{2}$ School of Systems Science, Beijing Normal University, Beijing, 100875, China
}

\begin{abstract}
Excitatory synaptic strength is reported to be positively related to the number of AMPARs in postsynaptic domain (PSD), which indeed actively transfer among postsynaptic density, extrasynaptic membrane (ESM), and dendritic cytosol. In this paper a biophysical model is developed to dissect the kinetics of AMPAR trafficking in equilibrium as well as during induction of synaptic plasticity. The analytical solutions are derived for both timescales of AMPAR exocytosisendocytosis and equilibrium distribution of AMPARs. Our model indicates differential roles of transition rates playing in inducing synaptic potentiation or depression, which include rate of AMAPR exocytosis, rate of AMPAR endocytosis, and AMPAR hopping rate between PSD and ESM region. In summary, our work highlights the underlying mechanism for AMPAR trafficking during dynamic equilibrium and synaptic plasticity, and provides mathematical insights that could be learned from to treat neurological diseases related to malfunctions of AMPAR movement.
\end{abstract}

\section{Introduction}

AMPA receptors mediate neurotransmitter transmission in excitatory synapses [1-4], which are activated during synaptic potentiation and depression in a protein-rich domain in postsynaptic membrane which is called postsynaptic density (PSD), and the number of AMPARs in PSD is believed to be proportional to the synaptic strength [5-7]. However, AMPARs are not static but continuously synthesized and transported into PSD as well as degenerated into intracellular cytosol. The trafficking process of AMPARs includes two basic processes: exocytosis and endocytosis. Experimental evidence reported that one way of AMPAR exocytosis is to firstly exocytosis into extra-synaptic membrane (ESM), and then diffuse into the PSD domain to react to the synaptic potentiation [8-11]; while the other exocytosis pathway shown in experimental observation is that AMPARs can directly insert into PSD domain from the intracellular receptor pools $[4,12,13]$. For the process of endocytosis of AMPARs, experimental results showed that AMAPRs appears to diffuse into ESM region where they are removed from the membrane, the direct endocytosis from PSD seems not be directly observed $[15,16]$. The precise mechanism including the molecular interactions for the exocytosis and endocytosis are under intensive investigation and are still not clear $[4,11,13,14]$.

Our work aims to dissect the kinetics of AMAPRs trafficking and provide possible pathways for specific AMPARs pathway causing synaptic potentiation and depression by computational modelling and mathematical analysis. We construct a "threecompartment" model to describe the transport of AMPAR exocytosis and endocytosis as well as diffusion. Specifically, our model consists of three compartments as PSD, ESM and intra-dendritic cytosol. The mathematical model describes the dynamics of the probability for AMPARS locating in each compartment. In detail, the model includes hopping process of AMPAR between ESM and the intracellular pools, i.e. AMPARs can be directly inserted into ESM, from where AMPARs can hop into PSD region. Besides, our model also incorporates the direct exocytosis of AMPARs into PSD with a different rate. However, for both cases, the AMPARs cannot directly endocytosis into cytosol according to the experimental observations, AMPARs are reported to firstly diffuse into the ESM region where they transfer into the cytosol $[15,16]$.

In the first section, we solve the mathematical equations of our model and obtain the equilibrium distribution of AMPARs in each compartment. Two timescales of AMPARs trafficking as functions of each rate parameters can be obtained, which are all functions of transition rates. We analyze the equilibrium distribution of AMPARs in PSD, ESM and cytosol as a function of different transition rates, and by changing each rate parameters our model demonstrates explicitly how AMPAR equilibrium distribution is regulated by exocytosis, endocytosis process, and the hopping process in simulation.

In the second section, we postulate possible pathways for induction of synaptic potentiation and depression by modulating AMPARs trafficking kinetics. Both equilibrium distribution and timescale of AMPARs in PSD region can be regulated by specific rate parameters. We change the corresponding parameters to test our hypothesis, and display the variation for each rate parameter 
needed in the induction of synaptic plasticity. This allows us to identify possible second-messenger pathways that could be responsible for the induction of long term potentiation (LTP) and long term depression (LTD).

\section{Model and methods}

Before introducing our model, we need to declare several assumptions made into our model as follows:

(a), the GluA1 and GluA2 are integrate elements of AMPAR, functioning as a one integrity, and we will not distinguish the function of each part;

(b), synthesis and degradation process are beyond our focus, and we only address the process of a population of AMPARs transporting among the three pools and how the specific group of AMPARs are dynamically regulated to distribute in PSD, ESM and dendritic cytosol;

(c), the exocytosis of AMPAR can occur both in spine PSD by the rate of $w_{a}$ and in ESM by the rate of $w_{b}$ for the case of $w_{a}=0$ the exocytosis only occurs in the ESM;

(d), the AMPARs in PSD are all active, we ignore the case of silent spine where AMAPR are not activated in PSD, meaning that the number of AMPARs represents the synaptic strength directly.

Several independent experimental observations tend to show that the AMPARs don't directly exchange between the PSD and dendritic cytosol. Instead, AMPARs exocytosis to the ESM area and then laterally diffuse into and out of the PSD area $[15,16]$. Besides, functional studies demonstrated that endocytosis of AMPARs occurs initially in ESM and the removal of AMPARs is by the lateral diffusion rather than direct removing from PSD, therefore, in our model we have the endocytosis process of AMPARs from ESM to cytosol only. For the diffusion process, we have the AMAPRs hopping between PSD and ESM. Considering that direct exocytosis of AMPAR vesicles into the postsynaptic membrane cannot be ruled out [5], in the exocytosis process, we have considered AMPARs insertion both into PSD and ESM with different rate parameters, and also compare the two cases to see how the differential exocytosis regulates AMPARs trafficking system in distinct ways.

\section{Three-compartment model: coupled ordinary differential equations for AMPAR trafficking}

We have constructed coupled differential equations for the dynamics of AMPAR trafficking in PSD $\left(p_{a}(t)\right), \operatorname{ESM}\left(p_{b}(t)\right)$ and dendritic cytosol $\left(p_{c}(t)\right)$ and the kinetics of the model is shown in the following equations:

$$
\left\{\begin{array}{c}
\frac{\mathrm{d} p_{a}(t)}{\mathrm{d} t}=-\frac{h}{A} p_{a}(t)+\frac{h}{A} p_{b}(t)+w_{a} p_{c}(t) \\
\frac{\mathrm{d} p_{b}(t)}{\mathrm{d} t}=\frac{h}{A} p_{a}(t)-\left(\frac{h}{A}+k\right) p_{b}(t)+w_{b} p_{c}(t) \ldots(1) \\
\frac{\mathrm{d} p_{c}(t)}{\mathrm{d} t}=k p_{b}(t)-\left(w_{a}+w_{b}\right) p_{c}(t) .
\end{array}\right.
$$

Where, $p_{a}$ represents the proportion of AMPARs in the PSD area, $p_{b}$ is the proportion of AMPARs in the ESM area, and $p$ the AMPARs in the dendritic cytosol, the total population of AMPARs is conserved by $p_{a}+p_{b}+p_{c}=1$. The parameter $\mathrm{A}$ is the extrasynaptic membrane area with dimension of $\mu m^{2}$; $h$ is the hopping rate per unit area between PSD and ESM with dimension of $\mu \mathrm{m}^{2} \mathrm{~s}^{-1} ; w_{a}$ is the exocytosis rate into PSD region with dimension of $s^{-1}$, and $w_{b}$ the rate of insertion into ESM region with dimension of $s^{-1}, K$ is the endocytosis rate of AMPARs from ESM into dendritic cytosol with dimension of $s^{-1}$.

\section{Analytical solutions for stationary state}

As time goes to infinity with $t \rightarrow \infty$, the trafficking system will approach dynamic equilibrium where the proportion of AMPARs in PSD, ESM and dendritic cytosol will not change with time. Mathematically, the equilibrium state solution can be obtained by setting

$$
\frac{\mathrm{d} p_{a}(t)}{\mathrm{d} t}=0 ; \frac{\mathrm{d} p_{b}(t)}{\mathrm{d} t}=0 ; \frac{\mathrm{d} p_{c}(t)}{\mathrm{d} t}=0 .
$$

We obtain the equilibrium state solution $p_{a}^{s}, p_{b}^{s}, p_{c}^{s}$ as follows:

$$
\begin{aligned}
& p_{a}^{s}=\frac{w_{a}(A k+h)+w_{b} h}{w_{b} h+w_{a}(A k+h)+h\left(w_{a}+w_{b}\right)+h k} \\
& p_{b}^{s}=\frac{2 w h h\left(w_{a}+w_{b}\right)}{w_{b} h+w_{a}(A k+h)+h\left(w_{a}+w_{b}\right)+h k} \\
& p_{c}^{s}=\frac{h k}{w_{b} h+w_{a}(A k+h)+h\left(w_{a}+w_{b}\right)+h k}
\end{aligned}
$$

As can be seen, in equilibrium state the proportion of AMPARs in each compartment depends on the rate parameters of $h, w_{a}, w_{b}, k$. Therefore, if these values are observed from experiments, our model could predict the exact proportion of the AMPARs in PSD, ESM and cytosol. On the other hand, if the experimental observation could tell the proportion of AMPARs in each compartment of PSD, ESM, and cytosol specifically, then our model could predict the values of rate parameters from the Eqn. (2).

At a special case, if there is no direct exocytosis of AMPARs from cytosol into PSD with $w_{a}=0$, then there will be only hopping process from ESM to PSD and vice versa with the same rate of $h$, and exocytosis from dendritic cytosol into only ESM.The equilibrium distribution of AMPARs will become:

$$
\left\{\begin{array}{l}
p_{a}^{s}=\frac{w_{b}}{2 w_{b}+k} \\
p_{b}^{s}=\frac{w_{b}}{2 w_{b}+k} \ldots \ldots \\
p_{c}^{s}=\frac{k}{2 w_{b}+k}
\end{array}\right.
$$

The proportion of AMPARs will be equal between PSD and ESM, and not depend on the hopping rate of $h$. This is a special case of the detailed balance solution. If the hopping rate from PSD to ESM (Say $h_{a}$ ) and from ESM to PSD (Say $h_{b}$ ) is different, then the distribution of AMPARs will become $p_{a}^{s} h_{a}=p_{b}^{s} h_{b}$.

\section{Analytical solution for exact dynamic evolution}

The eigenvalue for the coefficient matrix of coupled ordinary differential equation can be obtained by solving the determinant of coefficient matrix, and we have three solutions with $\lambda_{0}=0$ as follows:

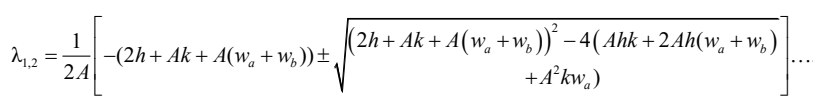

With the eigenvalues, the exact solution for the dynamics of AMPAR trafficking can be written as linear combination of the eigenvectors as follows:

$$
\left\{\begin{array}{l}
P_{a}(t)=A_{0}+A_{1} e^{\lambda_{1} t}+A_{2} e^{\lambda_{2} t} \\
P_{b}(t)=B_{0}+B_{1} e^{\lambda_{1} t}+B_{2} e^{\lambda_{2} t} \ldots \ldots . \\
P_{c}(t)=C_{0}+C_{1} e^{\lambda_{1} t}+C_{2} e^{\lambda_{2} t}
\end{array}\right.
$$


where, $A_{0}, A_{1}, A_{2}, B_{0}, B_{1}, B_{2}, C_{0}, C_{1}, C_{2} 9$ coefficients can be determined by the initial condition and the eigenvalues, the detailed procedure can be seen in the appendix. As seen above, once the initial condition $\mathrm{s}_{\mathrm{a}}$, $\mathrm{s}_{\mathrm{b}}$, $\mathrm{s}_{\mathrm{c}}$ are given, the coefficients of $C_{0}, C_{1}, C_{2}$ can be expressed explicitly as functions of $h, w, k$. With given coefficients of $C_{0}, C_{1}, C_{2}, A_{0}, A_{1}, A_{2}, B_{0}$, $B_{1}, B_{2}$ can be calculated out.

If we set $w_{a}=0$, then the timescale becomes:

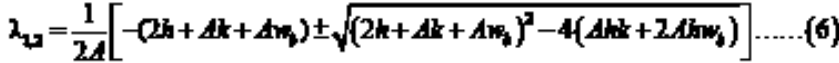

Which is dependent on the hopping rate $h$. We will show in the result section that it will be different in equilibrium distribution of AMPARs compared the case of $w_{a}=0.2778 s^{-1}$.

\section{Results}

\section{Equilibrium distribution of AMPARs and eigenvalues are independent of initial conditions}

Given the rates of parameters are chosen from [16]: $h=0.00125 \mu \mathrm{m}^{2} s^{-1} . w_{a}=w_{b}=0.2778 \mathrm{~s}^{-1}, k=0.000167 \mathrm{~s}^{-1}, A=0.1257 \mu \mathrm{m}^{2}$ the analytical expression for the equilibrium state Eqn. 2 can be calculated out as $p_{\mathrm{a}}^{\mathrm{s}}=0.6403, p_{b}^{s}=0.3492, p_{c}^{s}=0.01047$. And the eigenvalues $\lambda_{1}, \lambda_{2}$ by Eqn. 4 can be turned into two different timescales as: $\tau_{1}=\frac{1}{\left|\lambda_{1}\right|}=1.77 \mathrm{sec}, \tau_{2}=\frac{1}{\left|\lambda_{2}\right|}=35.4 \mathrm{sec}$.

We show that with different initial conditions of $s_{a}, s_{b}, s_{c}$, the equilibrium state approaches to the same values analytically predicted, which does not depend on the initial conditions of $s_{a}, s_{b}, s_{c}$. As can be seen in Figure 1A, where the initial condition is $s_{a}=0.0, s_{b}=0.5, s_{c}=0.5$ AMPARs in dendritic cytosol $(p)$ rapidly insert into ESM $(p)$ and PSD $\left(p_{a}\right)$, which corresponds to a rapid increase of AMPARs in PSD $\left(p_{a}\right)$, with timescales of $\tau_{1}=1.77 \mathrm{sec}$, the hopping process from ESM to PSD can also be seen from the decrease of $p_{b}$ and increase of $p_{a}$, which shows in a relatively slow timescale $\left(\tau_{2}=35.4 \mathrm{sec}\right)$. The dynamics eventually reaches an equilibrium state with $\mathrm{p}_{\mathrm{a}}^{\mathrm{s}}=0.6403, p_{b}^{s}=0.3492, p_{c}^{s}=0.01047$.

Similarly, in Figure 1B, where the initial condition is $s_{a}=0.1, s_{b}=0.7$, $s_{c}=0.7$ We can see that the AMPARs in dendritic cytosol sharply decreases (with timescale of $\tau_{1}=1.77 \mathrm{sec}$ ) while $p_{b}, p_{c}$, both increase in the similar speed as $p_{c}$, while the hopping process between, $p_{a}, p_{b}$, slows down the dynamics (with timescale of $\left(\tau_{2}=35.4 \mathrm{sec}\right)$ but eventually reaches the same equilibrium state with $p_{a}^{s}=0.6403, p_{b}^{s}=0.3492, p_{c}^{s}=0.01047$.

Not only the value but also the evolution of each population of AMPARs in PSD, ESM and dendritic cytosol are exactly the same between simulation and analytical solution Eqn. 6 .

In the following, we show how each process of exocytosis specifically regulates the dynamics of AMAPRs trafficking.

\section{AMPAR exocytosis regulates the equilibrium distribution and timescales of AMAPR trafficking}

\section{Exocytosis into ESM with rate $w_{b}$ regulates equilibrium distribution of AMPARs and timescales}

When there is no exocytosis into PSD with $w_{a}=0$ the equilibrium distribution of AMPARs in PSD and ESM is $p_{a}^{s}=p_{b}^{s}$ for all values of $w_{a}$, which has been shown by our model in Eqn. 4 and can be seen in Figure 2A. Both of $p_{a}^{s}$ and $p_{b}^{s}$ increase as $w_{b}$ increases sharply for small values of $w_{b}$. For instance, when $w_{b}=0.002778 s^{-1}$, we have $\mathrm{p}_{\mathrm{a}}^{\mathrm{s}}=p_{b}^{s}=0.12498, p_{c}^{s}=0.7500$, but when $w_{b}$ increased by 10 folds, we have $\mathrm{w}_{\mathrm{b}}=0.02778, p_{a}^{s}=p_{b}^{s}=0.3946, p_{c}^{s}=0.2308 p_{a}^{s}$ has increased by 3 folds; when $w_{b}$ increased by 20 folds, $w_{b}=0.05556 \mathrm{~s}^{-1}, p_{a}^{s}=p_{b}^{s}=0.4348, p_{c}^{s}=0.1304$. For even larger $w_{b}$, the distribution bare changes but eventually saturates at $p_{a}^{s}=p_{b}^{s}=0.4985, p_{c}^{s}=0.00029$ (as is shown in Figure 2A).

For the case of exocytosis process into PSD existing with $w_{a}=0.2778 s^{-1}$, the equilibrium distribution of AMPARs in PSD $p_{a}^{s}$ dramatically decreases with increasing $w_{b}$, while the AMAPRs in ESM $p_{b}^{s}$, dramatically increases with $w_{b}$, as can be seen in Figure 2C. For instance, when $w_{b}=0.002778 s^{-1}$. we have $p_{a}^{s}=0.7144, p_{b}^{s}=0.2695, p_{c}^{s}=0.016$; when $w_{b}$ increases by 10 times to $w_{b}=0.002778 s^{-1}$, we have $p_{a}^{s}=0.7046, p_{b}^{s}=0.2801, p_{c}^{s}=0.0153$. Further increase $w_{b}$ by 100 times to $w_{b}=0.2778 s^{-1}$, we have $p_{a}^{s}=0.6403, p_{b}^{s}=0.3492, p_{c}^{s}=0.0105$. Eventually increase $w_{b}$ by 1000 times to $w_{b}=2.778 s^{-1}$, the distribution becomes $p_{a}^{s}=0.5338, p_{b}^{s}=0.4634, p_{c}^{s}=0.00253$. When $w_{b}$ goes to extremely large, the final distribution of AMAPRs in PSD and ESM will approach the same value, as can be seen in Figure 2C. This indicates that when the exocytosis to ESM is extremely fast (much faster than other rates of $\left.w_{a}, h, k\right)$ this extreme large $w_{b}$ eventually transfers all the AMPARs into ESM; but with equal hopping rate $h$, the AMPARs will be equally distributed in PSD and ESM.

By comparing Figure $2 \mathrm{~A}$ and Figure $2 \mathrm{C}$, we can see that the equilibrium distribution of AMPARs in each compartment is totally different between the case of $w_{a}=0$ and the case of $w_{a}=0.2778 \mathrm{~s}^{-1}$. Especially when $w_{b}$ is small, exocytosis process into PSD $w_{a}$ plays a critically important role in promoting AMPARs into PSD, where they hop into ESM, leaving the AMAPRs in the dendritic cytosol with almost zero.

For all possible values of $w_{b}$ the AMPARs in PSD $p_{a}^{s}$ is always greater with $w_{a}=0.2778 s^{-1}$ than the case with $w_{a}=0$. Therefore we argue the direct exocytosis of AMPARs into PSD has greatly improved the synaptic efficacy during synaptic potentiation.

The timescales $\tau_{1}, \tau_{2}$ are regulated by the exocytosis rate $w_{b}$ differentially, as can be seen in Figure 2B and Figure 2D. For the case of no exocytosis into PSD with $w_{a}=0$ as is shown in the solid line in Figure $2 \mathrm{~B}$. For small values of $w_{b}$, the long time scale $\tau_{1}$ drops dramatically from $140 \mathrm{sec}$ at $w_{b}=0.002778 \mathrm{~s}^{-1}$, to $69 \mathrm{sec}$ at $w_{b}=0.02778 \mathrm{~s}^{-1}$, and eventually stabilize at $\tau_{1}=50.25 \mathrm{sec}$ afterwards. As for the second timescale of $\tau_{2}$, it drops from $\tau_{2}=30.64 \operatorname{secs}$ at $w_{b}=0.002778 \mathrm{~s}^{-1}$ to $\tau_{2}=20 \mathrm{secs}$ with $w_{b}=$ $0.02778 \mathrm{~s}^{-1}$, and further decreases to $\tau_{2}=3.389 \mathrm{secs}$ at $w_{b}=0.2778 \mathrm{~s}^{-1}$ and finally drops to $\tau_{2}=0.3578$ secs with $w_{b}=2.778 \mathrm{~s}^{-1}$, which can be seen in the dotted line in Figure 2B.

On the contrary, the long time scale is completely different for the case of exocytosis into PSD with rate $w_{a}=0.2778 \mathrm{~s}^{-1}$. For small values of $w_{b}$, the long time scale actually increases from $\tau_{1}=26.91 \mathrm{sec}$ with $w_{b}=$ $0.002778 \mathrm{~s}^{-1}$ to $\tau_{1}=28.11 \mathrm{sec}$ with $w_{b}=0.002778 \mathrm{~s}^{-1}$ and $\tau_{1}=35.46 \mathrm{sec}$ with $w_{b}=0.2778 s^{-1}$. Final increase $w_{b}$ by 1000 times to $w_{b}=2.778 s^{-1}$, we have $\tau_{1}=46.59 \mathrm{sec}$. As can we have the timescales of be seen in the solid line in Figure 2D. This increase of long time scale reflects that, with the exocytosis process into PSD existing, the rate increase of exocytosis into ESM $w_{b}$ actually slows down the system to become equilibrium in the long time scale.

As for the fast timescale $\tau_{2}$, it decreases from $\tau_{2}=3.57 \mathrm{sec}$ at $w_{b}=$ $0.002778 \mathrm{~s}^{-1}$ smoothly to $\tau_{2}=3.26 \operatorname{secs}$ with $w_{b}=0.02778 \mathrm{~s}^{-1}$, followed by $\tau_{2}=1.77 \mathrm{secs}$ with $w_{b}=0.2778 \mathrm{~s}^{-1}$. And eventually to $\tau_{2}=0.3256 \mathrm{secs}$ with $w_{b}=0.2778 s^{-1}$, as can be seen in dotted line in Figure 2D. From our analyses, the fast scale is almost the same with $0.35 \mathrm{sec}$ for extremely large rate of $w_{b}$ no matter the exocytosis into PSD happens or not.

In summary, by comparison of the timescales of the system between without exocytosis into PSD $w=0$ and with exocytosis into PSD with $w_{a}=0.2778 s^{-1}$, we can see that the exocytosis of AMPARs into PSD has greatly improved the efficiency of AMPAR trafficking system by shortening the longer timescale. Especially when $w_{b}$ is extremely small, the exocytosis process has an important function in driving the 

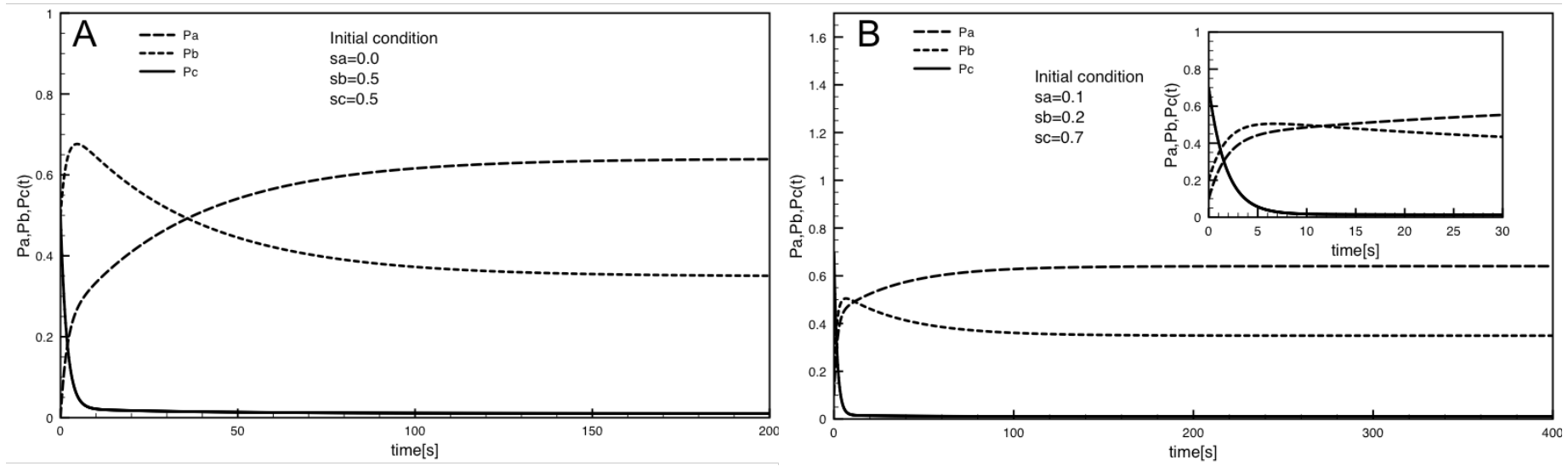

Figure 1: Equilibrium distribution of AMPARs does not depend on initial conditions. (A) The initial condition is $\mathrm{s}_{\mathrm{a}}=0.0, \mathrm{~s}_{\mathrm{b}}=0.5$, $\mathrm{s}_{\mathrm{c}}=0.5$. (B) The initial condition is $\mathrm{s}_{\mathrm{a}}=0.1, \mathrm{~s}_{\mathrm{b}}=0.2, \mathrm{~s}_{\mathrm{c}}=0.7$.
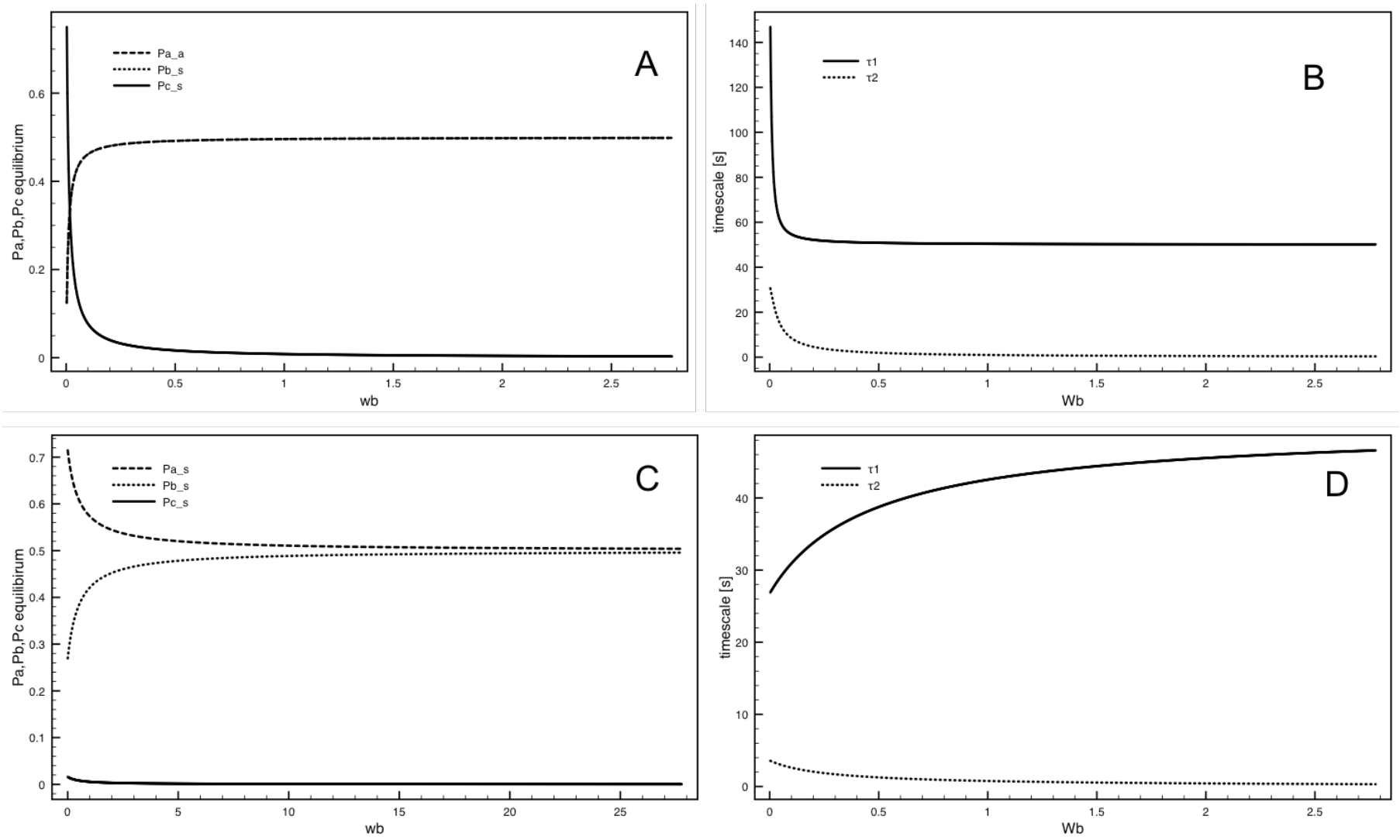

Figure 2: Exocytosis into ESM with rate $w_{b}$ regulates AMPAR distribution at equilibrium state and timescales. Distribution of AMPARs in equilibrium state when $w_{a}=0$ in (A) and $w_{a}=0.2778 s^{-1}$ in (C). Timescales change with $w_{b}$ when $w_{a}=0$ in (B) and $w_{a}=0.2778 s^{-1}$ in (D).

system approach equilibrium by a factor of 5 . When $w_{b}$ becomes close to $w_{a}$ or even greater than $w_{a}$, the function of $w_{b}$ has dominant role in determining timescales, regardless of $w_{a}$ on the timescales.

\section{Endocytosis rate $K$ from ESM into dendritic cytosol regulates AMPARs distribution at equilibrium state and eigenvalues}

In this section, we show that endocytosis process with rate $k$ regulates the equilibrium distribution of AMPARs and timescale of AMPARs trafficking in differential ways at two different conditions: one is $w_{a}=0, w_{b}=0.2778 s^{-1}$ by removing the exocytosis process into PSD; and the other is $w_{a}=w_{b}=0.2778 s^{-1}$ for both exocytosis into PSD and ESM. Note that there is a huge difference between the two case, we will illustrate the details in Figure 3.
When there is no exocytosis into PSD with $w_{a}=0$, the equilibrium distribution of AMPARs in PSD $p_{a}^{s}$ and ESM $p_{b}^{s}$ are equal, which both decrease with the increase of endocytosis rate $k$, as is shown in Figure $3 \mathrm{~A}$, with the dashed line $\left(p_{a}^{s}\right)$ and dotted line $\left(p_{a}^{s}\right)$ collides together, However, the proportion of AMPARs in cytosol $p_{c}^{s}$ increases with the increasing of the endocytosis rate $k$. AMPARs in PSD $p_{a}^{s}$ or $p_{b}^{s}$ does not change sensitively with $k$ when endocytosis rate $k$ is small, but decreases dramatically with large values of $k$, for instance, from 49.99 $\%$ to $12.49 \%$ when $k$ increases from $k=0.000167 \mathrm{~s}^{-1}$ to $k=1.67 \mathrm{~s}^{-1}$. This reflects the fact that the endocytosis has removed AMPARs from both ESM and PSD, which eventually transfers into dendritic cytosol.

When the trafficking process includes the exocytosis to PSD with $w_{a}=0.2778 s^{-1}$, the distribution of AMPARs in equilibrium state is totally different, as is shown in Figure 3C. The proportion of AMPARs 
in PSD goes opposite direction compared with it in ESM. When $k=0.000167 \mathrm{~s}^{-1}$, the system has the equilibrium state distribution as $p_{a}^{s}=0.5020, p_{b}^{s}=0.4978, p_{c}^{s}=0.0001494$. When $k$ increased 100 folds to $k=0.0167 \mathrm{~s}^{-1}$, the system has $p_{a}^{s}=0.6403, p_{b}^{s}=0.3492, p_{c}^{s}=0.01047$, notice that $p_{a}^{s}$ has increased 0.14 while $p_{b}^{s}$ has decreased 0.14 ; when $k$ increased to $k=0.167 \mathrm{~s}^{-1}$, the system has $p_{a}^{s}=0.8778, p_{b}^{s}=0.09403, p_{c}^{s}=0.02821$, where $p_{a}^{s}$ has increased by 0.375 and $p_{b}^{s}$ has decreased by $0.403, p_{c}^{s}$ has increased very few. Different to the situation with $w_{a}=0$, where the decrease of are going hand by hand with $p_{b}^{s}$; here the increase of AMPARs in PSD $p_{b}^{s}$ occurs simultaneously at the price of decrease of AMPARs in the ESM $p_{b}^{s}$ when increasing endocytosis rate $k$, while AMPARs in the dendritic cytosol $p_{c}^{s}$ barely changes.

By comparison the Figure $3 \mathrm{~A}$ and Figure $3 \mathrm{C}$, we can see that the process of direct exocytosis into PSD with rate $w_{a}$ keeps the AMPARs in PSD $p_{a}^{s}$ at a high value even with large endocytosis rate $k$, while in the case with no direct exocytosis $w_{a}=0$, the large endocytosis rate $k$ will keep $75 \%$ of the AMPARs in dendritic cytosol, leaving only 12.49\% AMPARs in both PSD and ESM. This fundamental difference demonstrates the importance of direct insertion of AMPAR into PSD.

The timescale of the dynamic system is regulated by the endocytosis rate $k$ differentially in the two cases, as can be seen in Figure 3B. For $w_{a}=0, w_{b}=0.2778 \mathrm{~s}^{-1}$ case, the long time scale increases from $\tau_{1}=50.03$ secs at $k=0.000167 \mathrm{~s}^{-1}$, to $\tau_{1}=62.11 \mathrm{secs}$ at $k=0.167 \mathrm{~s}^{-1}$, and eventually to $\tau_{1}=87.95 \mathrm{secs}$ at $k=1.67 \mathrm{~s}^{-1}$. While the short time scale decreases from $\tau_{2}=$ $3.598 \mathrm{secs}$ at $k=0.000167 \mathrm{~s}^{-1}$ to $\tau_{2}=2.23 \mathrm{sec}$ at $k=1.67 \mathrm{~s}^{-1}$, and eventually drops down to $\tau_{2}=0.5119 \mathrm{sec}$ at $k=0.167 \mathrm{~s}^{-1}$, The long time scale elongates while the short time scale drops down in this situation.

For the case having exocytosis process into PSD with $w_{a}=w_{b}=$ $0.2778 s^{-1}$ as can be seen in Figure 3D. For $k=0.000167 s^{-1}$, the system has the timescales of $\tau_{1}=50.00 \mathrm{secs}, \tau_{2}=1.79 \mathrm{secs}$. As $k$ has increased by 100 folds to $k=0.0167 \mathrm{~s}^{-1}$, the two eigenvalues becomes the timescales of $\tau_{1}=$ $35.71 \mathrm{secs}, \tau_{2}=1.77 \mathrm{secs}$. When endocytosis rate increases to $k=0.167 \mathrm{~s}^{-1}$, the timescales become $\tau_{1}=11.01$ secs, $\tau_{2}=1.534$ secs. When $k$ grows extremely large to $k=1.67 \mathrm{~s}^{-1}$, we have the timescales of $\tau_{1}=4.007 \mathrm{secs}, \tau_{2}=$ 0.5008 secs. Both the long time scale and fast time scale drops with the increase of endocytosis at this situation.

By comparison the Figure 3B and Figure 3D, when $w_{a}=0$, the long timescale gets longer and short timescale gets shorter when endocytosis rate $k$ goes up; while for the case of $w_{a}=w_{b}=0.2778 \mathrm{~s}^{-1}$, both long timescale and short timescale become shorter dramatically, especially in long timescale by 8 times. We can conclude hat, with all possible endocytosis rate $k$, the process of direct exocytosis into PSD has a crucial function in speeding up the dynamic system both in short timescale and long timescales.

\section{Dependence of equilibrium distribution of AMPARs and timescale on hopping rate $h$}

We have also investigated the equilibrium distribution and timescales change with hopping rate $h$, as can be seen in the simulation result Figure 4.

When there is no exocytosis into PSD occurred with $w_{a}=0$ the equilibrium distribution of AMPARs in each compartment is completely independent of hopping rate $\mathrm{h}$, as is shown in Figure 4A.

However, when there is exocytosis into PSD with $w_{a}=w_{b}=0.2778 s^{-1}$, the equilibrium distribution of AMPARs $p_{\mathrm{a}}^{\mathrm{s}}, p_{b}^{s}, p_{c}^{s}$ depends on the hopping rate $h$ of AMPARs between PSD and ESM, as is shown in Figure 4C. Besides, the way that the population of AMPARs in each compartment at equilibrium distribution changes with $h$ is totally
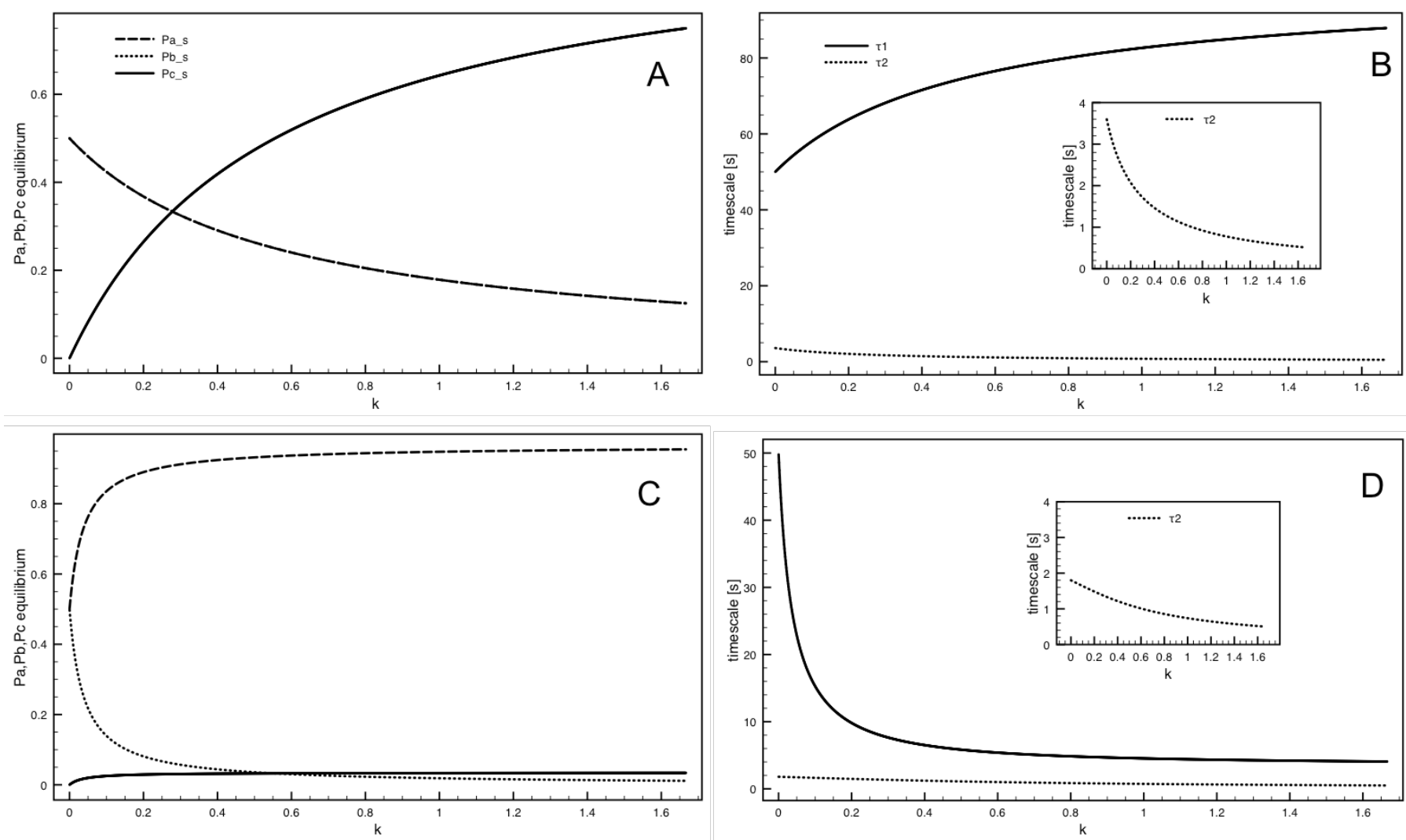

Figure 3: Compare k: Endocytosis rate $k$ regulates the equilibrium distribution of AMPARs and timescales. AMPARs distribution at equilibrium state depends on endocytosis rate $k$ for $w_{a}=0$ in (A) and $w_{a}=w_{b}=002778 s^{-1}$ in (C). The timescales depend on endocytosis rate $k$ for $w_{a}=0$ in (B) and $w_{a}=w_{b}=002778 s^{-1}$ in (D). The inner site plots show that the short timescale $\tau_{2}$ changes with $k$. 
different from the way of exocytosis rate and endocytosis rate $k$. The increase of hopping rate $h$ induces the decrease of population of AMPARs in PSD $p_{\mathrm{a}}^{\mathrm{s}}$, but with increase of population of AMPARs in ESM $p_{b}^{s}$, the population of AMPARs in dendritic cytosol $p_{\mathrm{c}}^{\mathrm{s}}$ is barely influenced by the change of hopping rate $h$.

As is shown in the Figure 4C, the proportion of AMPARs in PSD $p_{\mathrm{a}}^{\mathrm{s}}$ changes dramatically when $h$ is small with $h_{0}<0.01257 \mu \mathrm{m}^{2} s^{-1}$. For $h_{0}>0.01257 \mu \mathrm{m}^{2} s^{-1}$, there is no dramatic modulation of $h$ for the equilibrium distribution of AMPARs.

However, when $h$ increases by 100 times to $h_{0}=0.001257 \mu \mathrm{m}^{2} \mathrm{~s}^{-1}$, the equilibrium state has $p_{\mathrm{a}}^{\mathrm{s}}=0.6403, p_{b}^{s}=0.3492, p_{c}^{s}=0.0105$, showing that the population of AMPARs in PSD $p_{\mathrm{a}}^{\mathrm{s}}$ has decreased from $98 \%$ into $64 \%$, while the reduction of AMPARs in PSD has transferred into ESM $p_{\mathrm{a}}^{\mathrm{s}}$ from $9.64 \%$ to $34.92 \%$, the population of AMPARs in dendritic cytosol have not changed a lot. For even large $h$, i.e. $h_{0}=0.01257 \mu \mathrm{m}^{2} \mathrm{~s}^{-1}$, we have $p_{\mathrm{a}}^{\mathrm{s}}=0.5126, p_{b}^{s}=0.4732, p_{c}^{s}=0.01419$, showing that the population of AMPARs in PSD $p_{\mathrm{a}}^{\mathrm{s}}$ continues to drop to $51 \%$ and AMPARs in ESM $p_{b}^{s}$ continues to increase to $47.32 \%$ as the hopping rate $h$ increases, and both eventually stabilize near $50 \%$.

Both long timescale and short timescale decrease with increasing hopping rate for both the case with exocytosis into PSD as seen in Figure $4 \mathrm{~B}$ and the case without exocytosis into PSD as seen in Figure 4D. For $h<0.02 \mu \mathrm{m}^{2} \mathrm{~s}^{-1}$, long timescale decreases rapidly with the increase of $h$, while the short timescale does not change abruptly with the increasing of hopping rate $h$. For both situations in Figure 4B and Figure 4D, the short timescale eventually approaches similar value as hopping rate goes to extremely large. However, the timescales seem not change for the extreme high hopping rates. In general, both the long timescale and the short timescale for the system to approach equilibrium is shorter with the process of direct exocytosis into PSD with $w_{a}=w_{b}=0.2778 \mathrm{~s}^{-1}$. But with extreme high hopping rate, this timescale will not change.

\section{Regulation of AMPAR trafficking during synaptic plasticity}

We have shown in the previous section that each process of exocytosis, endocytosis and hopping activity affects the equilibrium distribution of AMPARs in PSD, ESM and dendritic cytosol as well as the timescale for the system to approach equilibrium. In this section, we further investigate how would each process of exocytosis, endocytosis and hopping process between PSD and ESM increase (decrease) the proportion of AMPARs in PSD during LTP (LTD), and how much modification would be made to induce the synaptic plasticity.

\section{Regulation of AMPAR trafficking during synaptic potentiation}

This investigation would provide a hint for experimental design to see which specific process would be most possible for the synapse to have physiological changes. To be illustrative, we set $w_{a}=w_{b}=w$ to make illustration simpler.

Firstly, increase of exocytosis rate $w$ can induce synaptic potentiation. As can be seen in Figure 5A, $w$ was set to be $0.1 w_{0}$ for the first $50 \mathrm{sec}$, where $w_{0}=0.2778 \mathrm{~s}^{-1}$; from $50 \mathrm{sec}$ to $250 \mathrm{sec}, w$ increased to $w_{0}$, the proportion of AMPARs in PSD $p_{a}(t)$ (dashed line) increases sharply from 0.31 to 0.63 by nearly $100 \%$, indicating a LTP process with $100 \%$ increase of synaptic strength. Simultaneously, the proportion of
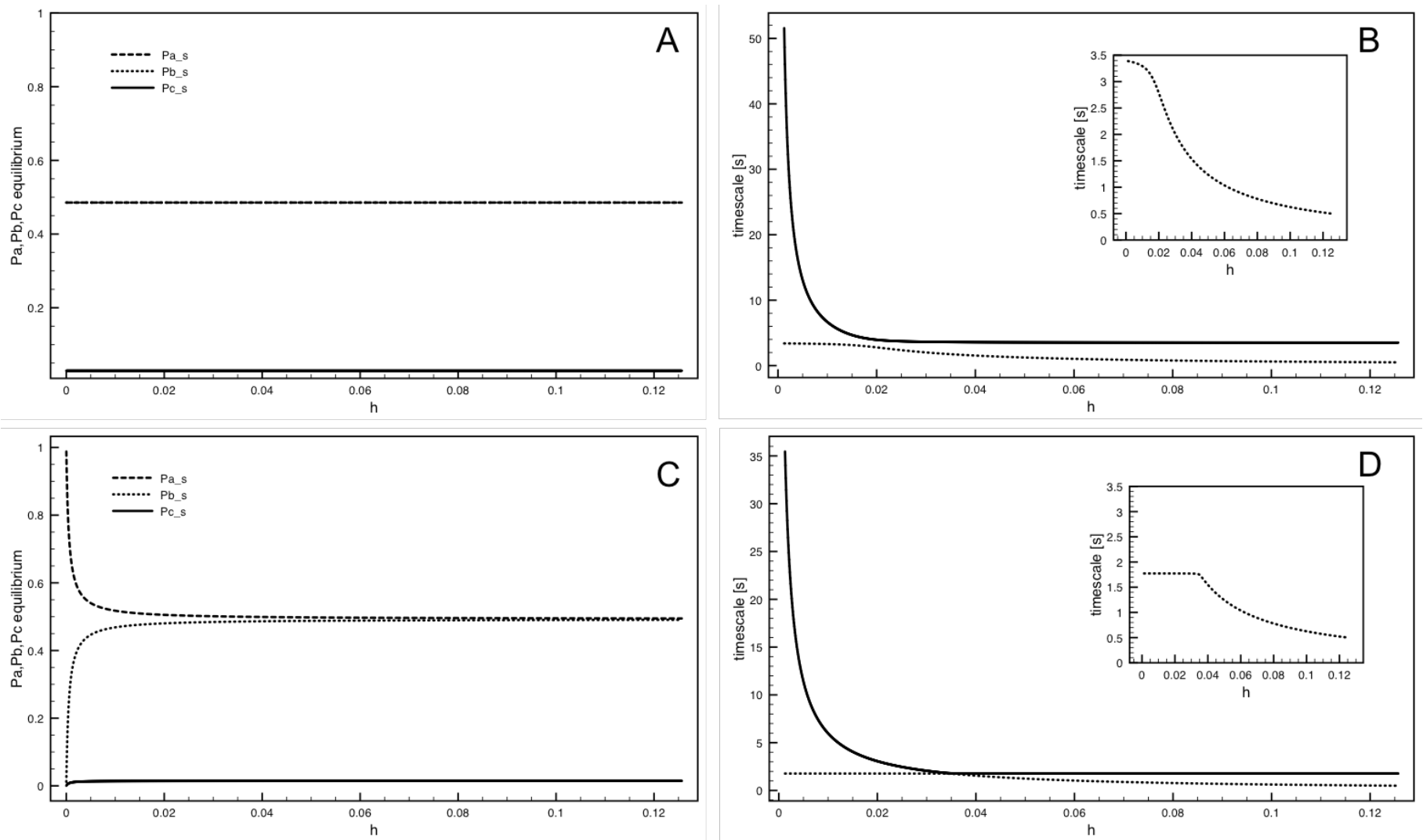

Figure 4: Compare $h$ : Hopping rate $h$ regulates the distribution of AMPARs at equilibrium state and timescales. A Equilibrium distribution of AMPARs depends on hopping rate of $h$ for $w_{a}=w_{b}=0.2778 s^{-1}$ in (A) and $w_{a}=w_{b}=0.2778 s^{-1}$ in (C). The timescales $\tau_{1}, \tau_{2}$ depend on rate parameter $h$ for $w_{a}=0, w_{b}=0.2778 s^{-1}$ in (B) and $w_{a}=w_{b}=0.2778 \mathrm{~s}-1$ in (D). The inner site plots show that the short timescale $\tau_{2}$ changes with $h$. 
AMPARs in $\mathrm{ESM}_{b}(t)$ (dotted line) increases as well, but the proportion of AMPARs in cytosol $p_{c}(t)$ (solid line) sharply decreases. It indicates that the AMPARs in dendritic cytosol provides the source of AMPARs to accumulate in both PSD $p_{a}(t)$ and $\operatorname{ESM}\left(p_{b}(t)\right)$. At $250 \mathrm{sec}$, we reset $w$ to the original value of $0.1 w_{0}$ as can be seen, the population of AMPARs in PSD returns to 0.31. As illustrated, the proportion of AMPARs in PSD $p_{a}$ can be doubled by up-regulation of exocytosis rate $w$ from $0.1 w_{0}$ to $w_{0}$ with 10 folds.

In addition, the increase of endocytosis rate $k$ leads to synaptic potentiation. As can be found in Figure 5B, the value of $k$ was set to be $0.01 k_{0}$ for the first $50 \mathrm{sec}$, where $k_{0}=0.167 \mathrm{~s}^{-1}$ From $50 \mathrm{sec}$ to $400 \mathrm{sec}$, the value of $k$ is set to be $k_{0}$, the population of AMPARs in PSD $p_{a}(t)$ increased from 0.3 to around 0.63 , corresponding to synaptic potentiation by 100 $\%$. Simultaneously, the proportion of AMPAR in ESM $p_{b}(t)$ drops from 0.49 to 0.35 , while the population of AMPARs in dendritic cytosol $p_{c}(t)$ only decreases very few. This change of AMPARs in each compartment indicates that the increased proportion of AMPAR in PSD $p_{a}(t)$ mainly comes from ESM $p_{b}(t)$ during synaptic potentiation, which is dramatically different from the case of modulation of exocytosis rate $w$ in Figure 5A

Another possible mechanism for induction of synaptic potentiation is to decrease of the hopping rate $h$. As can be seen in Figure 5C, the hopping rate $h$ has been initially set to be $h=h_{0}$ for the first $50 \mathrm{sec}$, where $h_{0}=0.001257 \mu \mathrm{m}^{2} \mathrm{~s}^{-1}$. From $50 \mathrm{sec}$ to $400 \mathrm{sec}$, the hopping rate was set as $h=0.1 h_{0}$, as is shown in the dashed line, there is a huge accumulation of AMPARs in the PSD compartment, and $p_{a}(t)$ has increased from 0.639 to be 0.968 , by $51 \%$ increase. Simultaneously the proportion of AMPARs in ESM $p_{b}(t)$ has correspondingly decreased from 0.3495 to 0.0342 . While the population of AMPARs in dendritic cytosol $p_{c}(t)$ bare changes. After $400 \mathrm{sec}$, we reset the hopping rate to be $h=h_{0}$, and the proportion of AMPARs in each compartment returns to the initial distribution. This demonstrate that increasing hopping rate $h$ can induce the synaptic potentiation, and the extent of synapse being potentiated is less than the modulation of either exocytosis rate $w$ or endocytosis rate $k$.

\section{Regulation of AMPAR trafficking during synaptic depression}

In correspondence to the increase of exocytosis rate $\mathrm{w}$, the reduction of $\mathrm{w}$ reduces the proportion of AMPARs in PSD, leading to synaptic depression. As can be seen in Figure 5D, the value of $\mathrm{w}$ was initially set to be $w_{0}$ for the first $50 \mathrm{sec}$. From $50 \mathrm{sec}$ to $250 \mathrm{sec}$, the rate parameter $w$ is set to be $0.1 w_{0}$. We can see that the proportion of AMAPRs in PSD $p_{a}(t)$ decreases from 0.585 to 0.35 by $40 \%$, indicating the synaptic strength has been reduced. Simultaneously, the proportion of AMPARs in ESM $p_{b}(t)$ decreases from 0.32 to 0.18 . The lost AMPARs in PSD and ESM are both transferred into the dendritic cytosol, as can be seen $p_{c}(t)$ sharply increases. At $250 \mathrm{sec}$, the value of $w$ was reset to be $w_{0}$, the population of AMPARs in PSD, ESM and dendritic cytosol returns to the initial distribution.

Decrease of endocytosis rate $k$ can reduce the proportion of AMPARs in PSD, leading to synaptic depression. As can be seen in Figure $5 \mathrm{E}$, the value of $k$ was initially set to be $10 k_{0}$ for the first $50 \mathrm{sec}$, and is decreased into $k_{0}$ from $50 \mathrm{sec}$ to $400 \mathrm{sec}$. As can be seen, due to decreased rate of endocytosis $k$, the proportion of AMPARs in PSD $p_{a}(t)$ has decreased indicating synaptic depression. The reduced AMPAR in PSD transferred into ESM, leading to increase of $p_{b}(t)$. And the population of AMPARs in dendritic cytosol $p_{c}(t)$ has also decreased. This dynamic process illustrate that, due to the slowing down of endocytosis rate $k$, a group of AMPARs accumulates in ESM. It is because the exocytosis process is going well as before, but the endocytosis process has been slowed down, therefore causing the accumulation of AMAPRs in ESM, and the system reaches another equilibrium state. After $400 \mathrm{sec}$, $k$ was reset to be $10 k_{0}$, and the trafficking system immediately return to the initial distribution corresponding to the reset rate parameters. How fast the system returns to the original state is determined by timescales of the system, which is around $1.7 \mathrm{sec}$.

Increase of hopping rate $h$ can reduce the proportion of AMPARs in PSD, leading to synaptic depression. As can be seen in Figure 5F, the hopping rate $h=0.1 h_{0}$ was initially set for the first $50 \mathrm{sec}$, and for $50 \mathrm{sec}$ to $400 \mathrm{sec}$, we set $h=h_{0}$, we can see that the $p_{a}(t)$ decreased from 0.90 to 0.6404 . The proportion of AMPARs in ESM $p_{b}(t)$ has accumulated into 0.3492 from 0.0964 . At $400 \mathrm{sec}$, we reset $h$ to be $h=0.1 h_{0}$, the AMAPRs returns into the equilibrium state. This demonstrates that the modulation of hopping rate $h$ only modulates the pools of AMPARs in PSD and ESM, without any influence of proportion of AMPARs in the cytosol.

\section{Comparison of potentiation and depression mechanism with no direct exocytosis into PSD}

In this section, the direct exocytosis into PSD is blocked by setting $w_{a}=0$, and the comparison of regulation of synaptic potentiation and depression between two conditions of $w_{a}=w_{b}=0.2778 \mathrm{~s}^{-1}$ is presented in the following.

For the induction of synaptic potentiation, the modulation of exocytosis into ESM with rate $w_{b}$ can be one way. As can be seen in Figure 6A, if $w_{a}=0$, for the first $50 \mathrm{sec}$, we set the population of AMPARs at equilibrium state for $w_{b}=0.01 w_{a}$, as $p_{a}^{s}=p_{b}^{s}=0.125, p_{c}^{s}=0.751$, then we increased $w_{b}$ from $0.01 w_{0}$ to $w_{0}$ in 50-250 sec, the population of AMPARs in PSD increases smoothly, while the AMPARs in ESM sharply increase due to the up regulation of $w_{b}$ by depleting AMPARs in dendritic cytosol, but eventually return to the equilibrium distribution. At $\mathrm{t}=250 \mathrm{sec}$, we return the $w_{b}$ back to the value of $0.01 w_{0}$, the distribution of AMAPRs returns back to the original one. Therefore, by increasing the rate of exocytosis into ESM $w_{b}$ the induction of LTP with accumulation of AMPARs in PSD results from transferring AMPARs from both dendritic cytosol and ESM .

On the contrary, if $w_{a}=0.2778 s^{-1}$, by the same modulation of $w_{b}$, the population of AMPARs in ESM $p_{b}^{s}$ increases by depleting the AMPARs in PSD $p_{a}^{s}$, leading to LTD, as can be seen in Figure 6B. Therefore, the same modulation of $w_{a}$ leads to completely different synaptic activities, depending on the occurrence of direct exocytosis into PSD.

The similar phenomenon applies to the synaptic changes by modulation of endocytosis rate $k$. For period of 50-250s, the endocytosis rate $k$ is decreased from $10 k_{0}$ to $k_{0}$ then at $250 \mathrm{sec}$, the value is reset back into $10 k_{0}$. As can be seen in Figure 7A, for $w_{a}=0$, the decrease of endocytosis rate $k$ leads to increase of AMPARs in both PSD $p_{a}^{s}$ and ESM $p_{b}^{s}$, by depleting the AMPARs in the dendritic cytosol, resulting in LTP. On the contrary, the same modulation of endocytosis rate $k$ leads to opposite result in Figure 7B, where the direct exocytosis into PSD is set with rate $w_{a}=0.2778 s^{-1}$, the decrease of endocytosis rate $k$ leads to decrease of AMAPRs in PSD $p_{a}^{s}$ but increase of AMPARs in ESM $p_{b}^{s}$, leading to LTD.

Different synaptic activities can be induced by modulation of hopping rate $h$ depending on the direct exocytosis into PSD with rate $w_{a}$. As can be seen in Figure 8A, for $w_{a}=0$, even though $p_{a}(t)$ decreases, still the equilibrium solution $p_{a}^{s}$ does not change but with a faster timescale. If we set the initial condition as the equilibrium distribution, there will be no change of $p_{a}^{s}, p_{b}^{s}, p_{c}^{s}$ when modulating the hopping rate $h$. However, as can be seen in Figure 8B, where $w_{a}=0.2778 s^{-1}$, up-regulating the hopping rate $h$ decreases equilibrium distribution of AMPARs in PSD $p_{a}^{s}$, from which the AMPARs transfer to ESM resulting in LTD. 

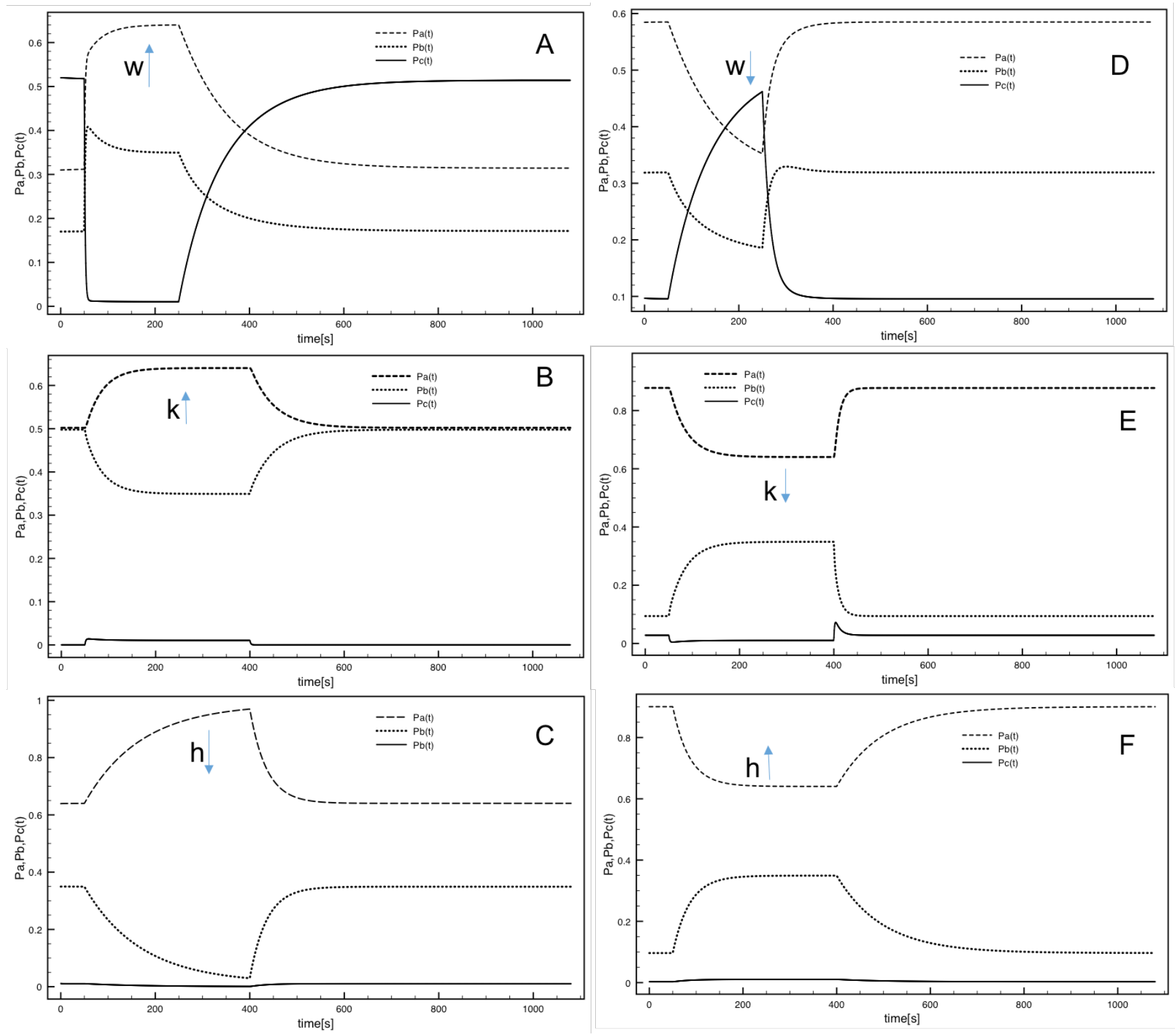

Figure 5: LTP and LTD can both be induced by modulating the endocytosis and exocytosis, hopping rates. A, B, C are for synaptic potentiation process; while D, E, $\mathrm{F}$ are for synaptic depression process.
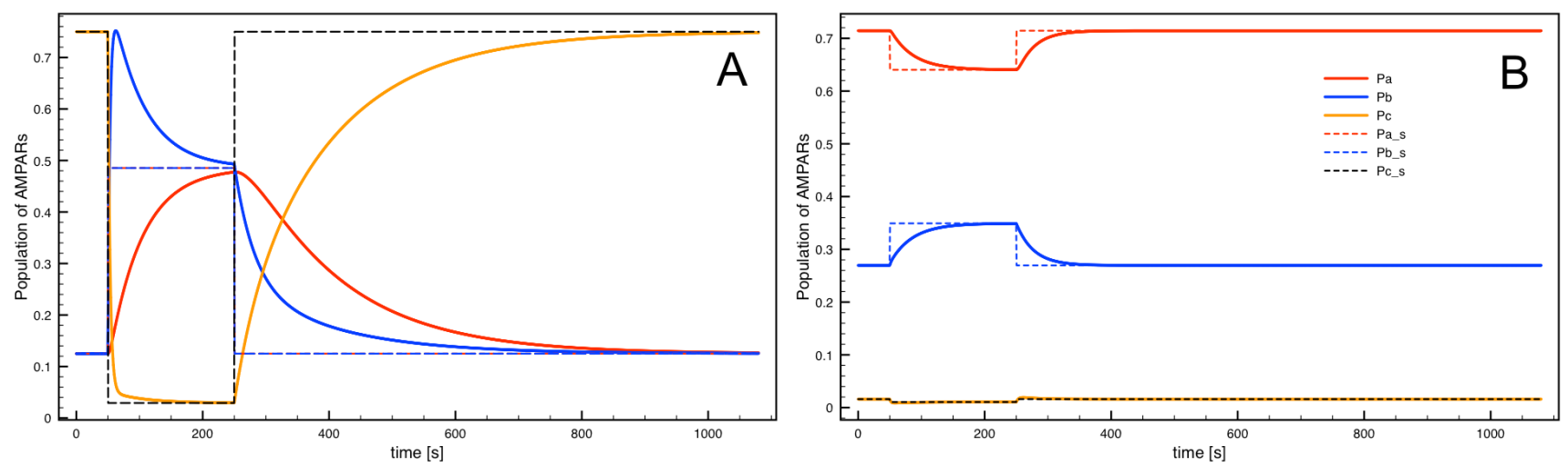

Figure 6: By increasing $w_{b}$ from $0.01 w_{0}$ to $w_{0}$ in $[50,250] \mathrm{sec}$, for (A) $w_{a}=0$, LTP is induced, while for (B) $w_{a}=0.2778 s^{-1}$, LTD is induced. 
In conclusion, whether there is a direct exocytosis into PSD is critically important for modulating each rate parameters to reach desired changes of synaptic strength. Based on this, one could propose that there is a stochastic process that AMAPRs can insert directly into PSD with random events, which can be considered as two basic working modules: one is with no direct exocytosis into PSD, the other is with direct exocytosis into PSD, the realistic complex AMPARs trafficking system can be described by a random process or more sophistic stochastic processes. Therefore, modulations of each rate parameters might lead to completely different synaptic activities, depending on how much composition of each two working module occupies and the timing between them to switch on working.

\section{Discussion and conclusion}

\section{Comparison to other models: what's new in our model}

We have considered two cases of exocytosis process in our model: one is with the direct exocytosis into PSD and the other is without direct exocytosis into PSD.

We found the big difference is that the process of direct exocytosis into PSD has increased proportion of AMPARs in PSD by nearly $50 \%$ compared with the case without the direct exocytosis, and this proportion of AMPARs raised in PSD is compensated by the reduction of AMPARs in the ESM; but when exocytosis into PSD rate $w_{a}$ is approaching the rate into ESM $w_{b}$, the final distribution of AMPARs into PSD, ESM and cytosol will not change, indicating that there is a specific value for rate $w_{a}$ to be saturated for the AMAPRs in PSD.

The regulation of AMPAR trafficking by the endocytosis rate $k$ has different results at the two different conditions. When there is no direct exocytosis into PSD $w_{a}=0$, the equilibrium distribution of AMPARs in PSD $p_{a}^{s}$ and ESM ${ }^{a} p_{b}^{s}$ are equal, both of them decrease when endocytosis rate $k$ gets larger. When there is direct exocytosis with rate $w_{a}=0.2778 s^{-1}$, the AMPARs in PSD has increased but the AMPARs in ESM $p_{b}^{s}$ has decreased along with the increase of endocytosis rate $k$.

When there is no direct exocytosis into PSD $w_{a}=0$, the hopping process with rate $h$ has no affect on the equilibrium distribution of AMPARs in PSD $p_{a}^{s}$ and ESM $p_{b}^{s}$ and $p_{a}^{s}=p_{b}^{s}$ for all possible values of $h$. However, when there is direct exocytosis into PSD with rate $w_{a}=0.2778 s^{-1}$, the AMPARs in PSD $p_{a}^{s}$ decreases sharply along the increase of $h$ but AMPARs in ESM $p_{b}^{s}$ increases dramatically with $h$ increases.

Our model showed that during synaptic activation, the synaptic potentiation and depression could be caused by each of changes in the exocytosis rate $w$, endocytosis rate $k$ and hopping rate $h$. Not only for the equilibrium distribution of AMPARs, but also for the timing to approach new equilibrium distribution, our model provided detailed calculation in mathematical analysis. From the expression of ratedependent equilibrium distribution, we could up-regulate or downregulate either rate parameter with specific modification to reach
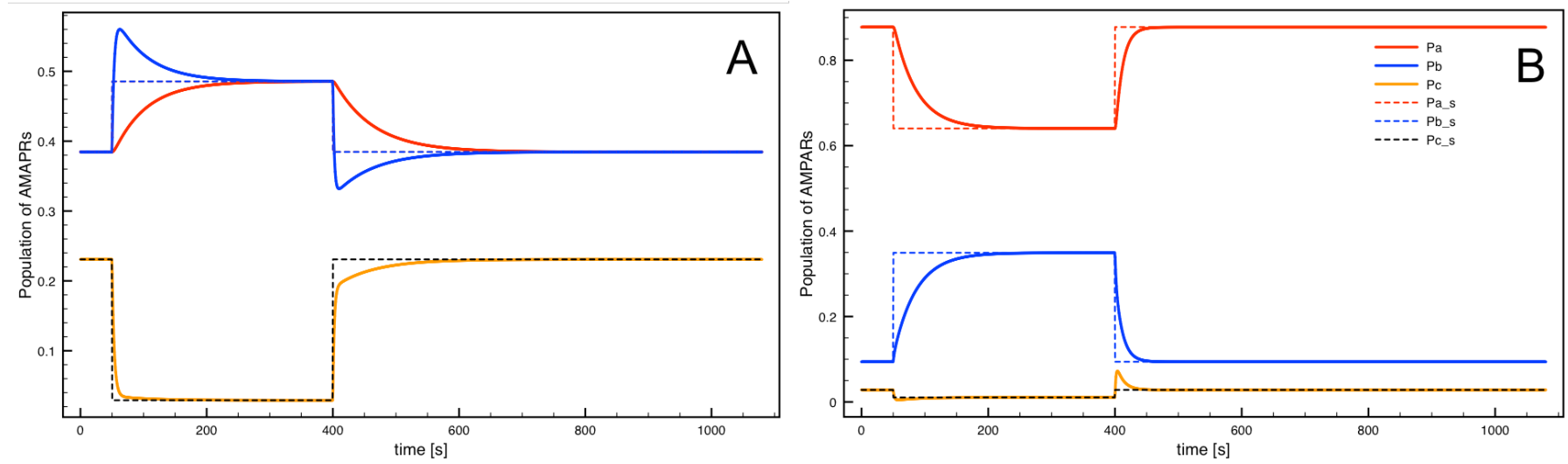

Figure 7: By decreasing endocytosis rate $k$ from $10 k_{0}$ to $k_{0}$ during $[50,250] \mathrm{sec}$, for (A) $w_{a}=0$, LTP is induced, but for (B) $w_{a}=0.2778 s^{-1}$, LTD is induced.
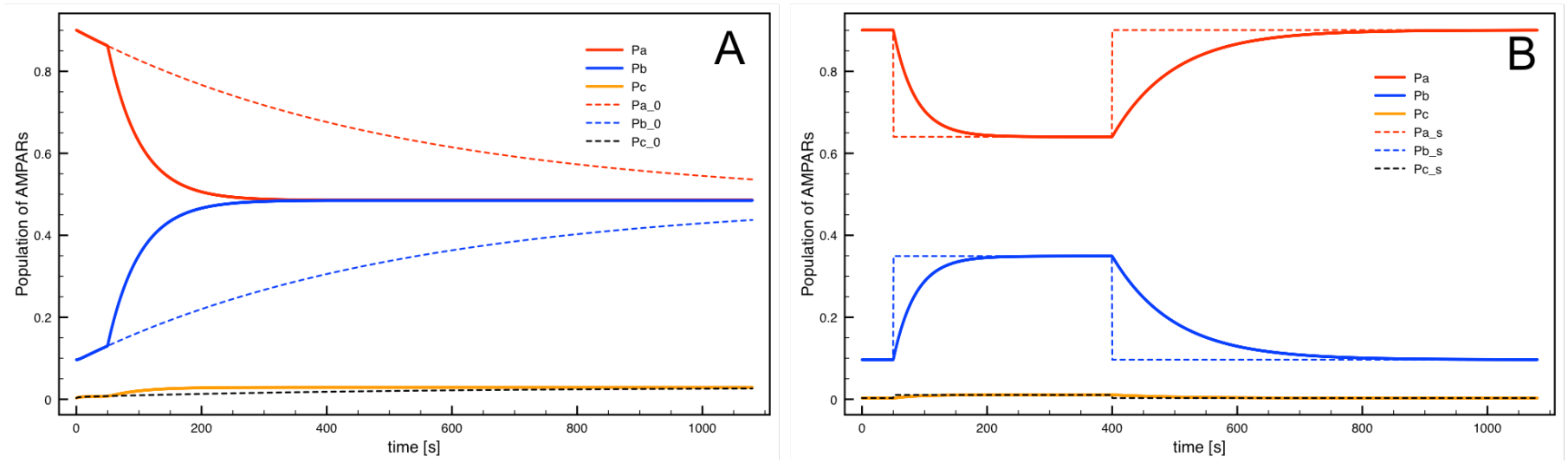

Figure 8: By increasing hopping rate $h$ from $0.1 h 0$ to $h 0$ during $[50,250] \mathrm{sec}$, for $(\mathrm{A}) w=0$, AMPARs in PSD is decreased but the equilibrium distribution is not changed; for (B) $w_{a}=0.2778 s^{-1}$, LTD is induced. 
the desired distribution of AMPARs in PSD. Therefore we provide a promising mathematical tool for experiments to test all possible pathways for synaptic potentiation or depression-related AMAPRs trafficking system. Besides, our model also provides the timescale to reach the final distribution, and the timing for AMPARs transferring to each compartment will finally determine how long one synapse needs to get potentiated or depressed.

\section{Calcium in mediating synaptic potentiation and depression and downstream signaling pathway}

The calcium influx can also trigger CaMKII and PKC pathway which eventually move AMAPRs into PSD, either through diffusion pathway or through direct exocytosis. NAMDR-dependent LTP is induced by $\mathrm{Ca}^{2+}$ influxes through NMDAR channels; another form of LTP is induced by L-type $\mathrm{Ca}^{2+}$ channels independent of presynaptic neurotransmitter release or postsynaptic NMDARs. Experimental evidence shows that postsynaptic Syt1- Syt7, the loss of which blocks LTP induced by postsynaptic increases in $\mathrm{Ca}^{2+}$ independent of the source of $\mathrm{Ca}^{2+}$ [5], and this Syt1-Syt7 loss impairs AMPAR exocytosis. Both proteins function as calcium sensors for AMAPR exocytosis.

Multiple signaling pathways can induce AMPA receptor internalization, calcium influx is specifically studied in the experiments. It is reported that the endocytosis of AMPARs is important for the expression of long-term depression triggered by NMDA receptor activation, which showed that the NMDA receptors alone can trigger AMPA receptor endocytosis through calcium influx and activation of the calcium-dependent protein phosphatase calcineurin. Calcium influx would probably influence several chemical reactions simultaneously through multiple pathways, for instance, during synaptic potentiation, the calcium-induced enzyme reactions may increase the exocytosis rate of AMPRS into ESM and PSD on one hand, and on the other hand, it may also decrease the hopping process of AMPARs between PSD and ESM.

Our model provides several path way but does not rule out the possibility of multiple pathway interact with each other and work coherently to play a function during synaptic potentiation and depression. From our simulation result of the synaptic potentiation and depression process, we predict that each rate parameters could be written as certain function of calcium density. The exocytosis rate $w$ is probably an increasing function of calcium levels, and the endocytosis rate parameter $k$ is also a mono-increasing function of the calcium level; while the hopping rate $h$ is a decreasing function of calcium level. The question of how exactly calcium influx modulates the exocytosis and endocytosis rate parameters and hopping rate is due to the future investigation.

\section{Acknowledgments}

This research is supported by the China Science Foundation for Young scholars and Central University research fund.

\section{References}

1. Penn AC, Zhang CL, Georges F, Royer L, Breillat C, et al. Hippocampal LTP and contextual learning require surface diffusion of AMPA receptors. Nature. 2017; 1: 1-10.

2. Makino H, Roberto Malinow R. AMPA receptor incorporation into synapses during LTP: the role of lateral movement and exocytosis. Nature. 2009; 64: 389-390.

3. Shepherd JD, Hunganir RL. The Cell Biology of Synaptic plasticity: AMPA receptor trafficking. Annu Rev Cell Biol. 2007; 23: 613-643.

4. Malinow R, Malenka RC. AMPA receptor trafficking and synaptic plasticity. Annu Rev Neurosci. 2002; 25: 103-126.

5. Wu D, Bacaj T, Morishita W, Goswami D, Arendt KL, et al. Postsynaptic synaptotagmines mediate AMPA receptor exocytosis during LTP. Nature. 2017; 544: 316-321.

6. Beattie EC, Carroll RC, Yu X, Morishita W, Yasuda H, et al. Regulation of AMPA receptor endocytosis by a signaling mechanism shared with LTD. Nature. 2003; 3: 1291-1300.

7. Passafaro M, Pech V, Morgan Sheng M. Subunit-specific temporal and spatial patterns of AMPA receptor exocytosis in hippocampal neurons. Nature Neurosci. 2003; 4: 917-926.

8. Earnshaw BA, Bressloff PC. Biological model of AMPA receptor trafficking and its regulation during long-term potentiation/long-term depression. J Neurosci. 2006; 26: 12362-12373.

9. Bredt DS, Nicoll RA. AMPA receptor trafficking at excitatory synapses. Neuron. 2003; 9: 361-379

10. Kessels HW, Malinow R. Synaptic AMPA receptor plasticity and Behavior Nature. 2009; 61: 340-350.

11. Roth RH, Zhang Y, Huganir RL. Dynamic imaging of AMPA receptor trafficking in vitro and in vivo. Current Opinion Neurobiology. 2017; 45: 51-68.

12. Santos SD, Carvalho AL, Caldeira MV, Duarte CB. Regulation of AMPA receptors and synaptic plasticity. Neurosci. 2009; 158: 105-126.

13. Chater TE, Goda Y. The role of AMPA receptors in post synaptic mechanisms of synaptic plasticity. Frontiers Cellular Neurosci. 2014; 8 1-14.

14. Anggono V, Huganir RL. Regulation of AMPA receptor trafficking and synaptic plasticity. Current Opinion Neurobiology. 2012; 22: 461-469.

15. Michael C. Ashby, Sarah A. De La Rue, et.al. Removal of AMPA receptors from synapses is preceded by transient endocytosis of extrasynaptic AMPARs. J Neurosci 2014; 24: 5172-5176.

16. Opazo P, Choquet D. A three-step mode of the synaptic recruitment of AMPA receptors. Mol Cellular Neurosci. 2011; 46: 1-8. 